\title{
PNET “ONDM 2019” special issue
}

\author{
Anna Tzanakaki ${ }^{1} \cdot$ Emmanouel Varvarigos $^{2} \cdot$ Raul Muñoz $^{3} \cdot$ Reza Nejabati $^{4} \cdot$ Noboru Yoshikane $^{5} \cdot$ \\ Markos Anastasopoulos ${ }^{4}$
}

Received: 6 November 2020 / Accepted: 6 November 2020 / Published online: 24 November 2020

(c) Springer Science+Business Media, LLC, part of Springer Nature 2020

\section{Introduction}

This PNET ONDM 2019 special issue (SI) includes extended versions of 14 papers, out of 55 in total oral and poster papers presented at ONDM 2019, held in Athens, Greece, in May 2019. These papers were selected and invited to be submitted to this SI based on their high-quality content. The papers focus on cutting-edge research in established areas of optical networking as well as their adoption in support of a wide variety of new services and applications. This involves the most recent trends in core, metro and access networks including elastic optical networks, software platforms relying on software-defined networking (SDN), network function virtualization (NFV), 5G and beyond, cloud/edge computing, artificial intelligence-assisted networks, secure and resilient networks as well as data center networks. All submitted papers went through a thorough peer-review process, and their content is briefly summarized below.

In paper Virtualized Controller Placement for MultiDomain Optical Transport Networks using Machine Learning, a dynamic controller placement method for optical transport networks is proposed. This takes into consideration constraints such as heterogeneity of optical controllers and resource limitations at the edge hosting locations and exploits a machine learning framework to support proactive prediction for the controller placement.

The paper Experimental Evaluation of Dynamic Resource Orchestration in Multi-Layer (Packet over Flexi-Grid Optical) Networks compares two online cloud and network

Anna Tzanakaki

atzanakaki@phys.uoa.gr

1 National and Kapodistrian University of Athens, Athens, Greece

2 National Technical University of Athens, Athens, Greece

3 CTTC, Barcelona, Spain

4 University of Bristol, Bristol, UK

5 KDDI Research Inc., Fujimino, Japan resource allocation algorithms used to dynamically accommodate network services within distributed remote interconnected data centers. The proposed algorithms are experimentally validated and compared on the CTTC ADRENALINE testbed.

The paper Evolving Optical Interconnection Topology: from Survivable Rings to Resilient Meshes proposes the use of twin graph network topologies with the aim to offer intrinsic resilience, as they satisfy equal length disjoint paths with minimal number of physical links and can graciously scale up/down and support merge/split topologies.

The paper Network Coding-based Routing and Spectrum Allocation in Elastic Optical Networks for Enhanced Physical Layer Security proposes an eavesdropping-aware routing and spectrum allocation approach utilizing network coding in elastic optical networks as well as a heuristic approach that solves the combined network coding, routing and spectrum allocation problem.

The paper A Novel Carrier-Cooperation Scheme with an Incentive to Offer Emergency Lightpath Support During Disaster Recovery proposes the use of a novel planning scheme enabling cooperation between carriers to achieve fast recovery of optical transport networks in case of disasters.

The paper Dynamic Wavelength and Bandwidth Allocation for Supporting Diverse Customers and Prioritized Traffic in NG-PON2 Networks focuses on resource allocation in PONs and more specifically proposes five different dynamic wavelength and bandwidth allocation schemes. The proposed approaches are evaluated through the development of heuristic algorithms based on insights from integer linear programming models that have been also developed with the aim to minimize the total delay of the high-priority data.

The paper Adaptive FH optimization in MEC-assisted $5 G$ environments proposes the use of evolutionary game theory to allow dynamic identification of the optimal split option in centralized radio access networks combining both the mobile edge computing model and relatively large-scale centralized data centers. In addition, optimal placement of 
the SDN controllers is determined adopting a heuristic algorithm that guarantees the stability of the whole system.

The paper Real-time Maintenance of Latency-sensitive $5 G$ Services through Network Slicing exploits the use of network slicing and focuses on the deployment and maintenance of services which are sensitive to latency constrains. In this context, a multi-segment architecture is presented along with a mechanism for latency sensing deployed in the service chain in the form of a VNF.

The paper Optimized placement of virtualized resources for $5 G$ services exploiting live VM migration proposes a heuristic algorithm suitable for BBU functions allocation in centralized softwarized radio access networks and evaluates the impact of dynamic resource management in these environments, facilitated through virtual machine (VM) live migration.

The paper Dedicated and Shared Solutions for Reliable Function Splitting in $5 G X$-haul paper proposes novel reliable function placement algorithms suitable for functional splitting, in 5G X-haul networks. These aim to guarantee service continuity in case of single central unit/distributed unit or link failure, based on dedicated and shared path protection principles.

The paper Macroscopic and microscopic techno-economic analyses highlighting aspects of $5 G$ transport network deployments provides insights of $5 \mathrm{G}$ transport network technologies and deployments as retrieved through macroscopic and microscopic techno-economic analyses and demonstrates their applicability on the architectural concepts of the 5G-PPP project 5G-PICTURE.

The paper System Innovations in Open WDM DCI Networks summarizes the evolution and innovations of data center interconnection transport systems deployed today. In this context, important enablers are the state-of-the-art coherent transmission exceeding $6 \mathrm{~b} / \mathrm{s} / \mathrm{Hz}$ in transatlantic deployments and software innovations in automation and programmability simplifying operation and enabling the emergence of "open" transport architectures.

The paper Virtual Network Mapping in Elastic Optical Networks with Sliceable Transponders focuses on the static version of the virtual network mapping problem in elastic optical networks equipped with sliceable bandwidth variable transponders with the objective to minimize the total transponder cost in the network.

The paper Hybrid and Optical Packet Switching Supporting Different Service Classes in Data Center Networks investigates different data center network solutions and identifies the hybrid switch approach as the approach to boost the performance of the data center network. Furthermore, the introduction of a "Reliable" service class proved to improve the performance of this class for the case of both the hybrid and the optical switch.
Publisher's Note Springer Nature remains neutral with regard to jurisdictional claims in published maps and institutional affiliations.

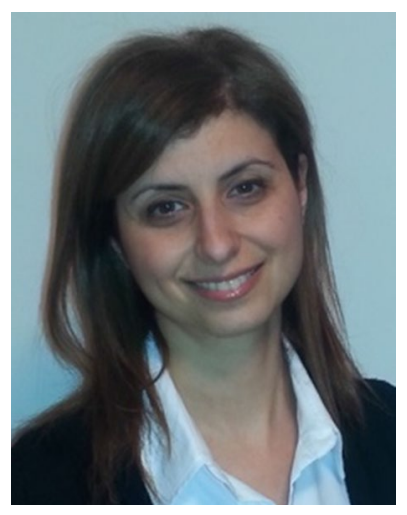

Anna Tzanakaki is an Associate Professor at the National and Kapodistrian University of Athens, Greece, and a Visiting Research Fellow at the University of Bristol, UK. She was previously an Associate Professor at Athens Information Technology, Greece, and an adjunct faculty member of the Information Networking Institute of Carnegie Mellon University, USA. She was a co-founder and a senior engineer of Ilotron Ltd., a hightechnology spin-off from the University of Essex. She is a coauthor of over 200 publications and a co-inventor of several patents. She is a Technical Programme Committee member of numerous international conferences and was the general chair of ONDM 2019. She has participated in a number of European and national research projects and is the Technical Coordinator of the EU 5G PPP Phase 2 project 5G PICTURE and Phase 3 project 5G-VICTORI.

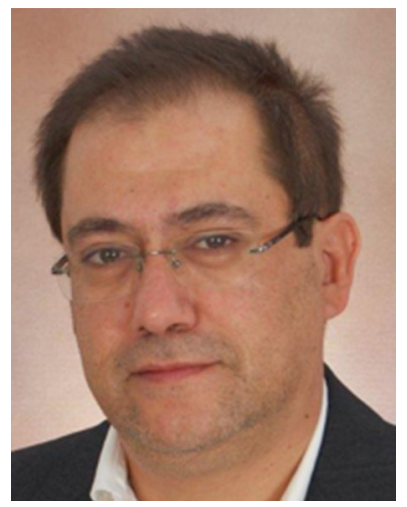

Emmanouel Varvarigos received a Diploma in ECE from NTUA, Greece, and the M.S. and Ph.D. degrees in EECS from MIT, USA. He has held faculty positions at UCSB, Delft University of Technology, University of Patras and Monash University, where he was also the Head of ECSE Department. He is currently a Professor at the ECE department of the National Technical University of Athens. He is a member of the board of the Computer Technology Institute-Diophantus (CTI), and Scientific Director of the Greek School Network division of CTI. He has participated in more than $40 \mathrm{US}$, Australian and EU-funded research projects in the areas of optical networking, smart energy grids and cloud computing, as well as in many national research projects, and has been the Consortium Coordinator in 10 of them. He has over 400 publications in refereed international journals and conferences.

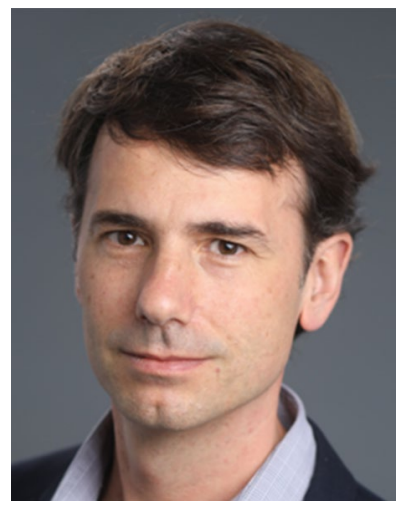

Raul Muñoz is graduated in Telecommunications Engineering and received a Ph.D. degree, both from the Universitat Politècnica de Catalunya (UPC), Spain. He is the Head of the Optical Networks and Systems Department at CTTC. He has participated in $50 \mathrm{R} \& \mathrm{D}$ projects funded by EC, Spanish Research programs and industrial contracts. He has been the Project Coordinator of the FP7 EUJapan STRAUSS project and the H2020-MSCA-ITN ONFIRE 
project. He has served as a General Chair of ONDM 2020 and WWRF39 and TPC chair of ECOC 2015 and ONDM2019. He was an academic member of the 5G IA Board, the Steering Board of WWRF and the Networld2020 ETP (2015-2017). He has published over 85 JCR journal papers, 275 international conference papers and 3 patents. He has been an IEEE Senior Member since 2012.

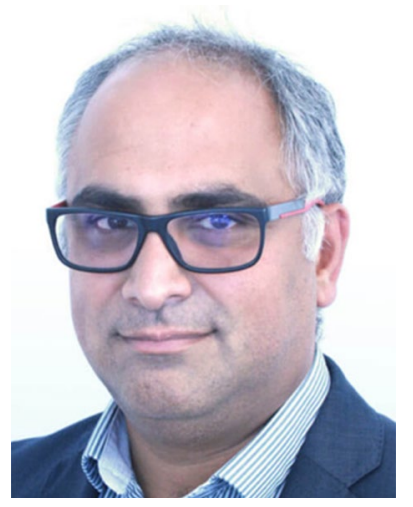

Reza Nejabati is currently a chair professor of intelligent networks and the head of High-Performance Network Group in the Department of Electrical and Electronic Engineering in the University of Bristol, UK. He has established internationally recognized research activities on "Autonomous and Software Defined Network" and "Quantum Network." His research received the prestigious IEEE Charles Kao Award in 2016 and has important contributions in $5 \mathrm{G}$, smart city, quantum communication and future Internet experimentation. He played a key role in development and success of four flagship national test beds, i.e., Bristol is Open Smart City test bed, 5G UK test bed, National Dark Fibre Facility and UK Quantum Network. Building on his research, he cofounded a successful start-up company (Zeetta Networks Ltd.) with 25 employees and $£ 6 \mathrm{M}$ VC and external funding.

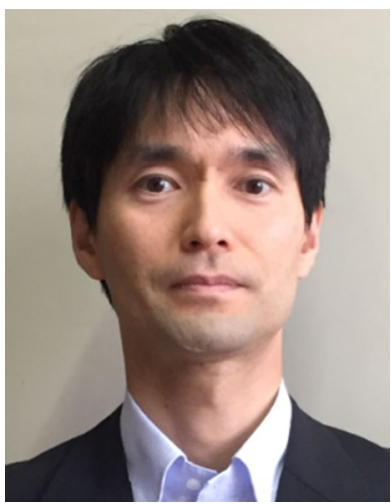

Noboru Yoshikane is a member of KDDI Research, Inc., Japan, focusing on future optical network technologies. He received his B.E., M.E. and Ph.D. degrees from Kyushu University, Fukuoka, Japan, in 1997, 1999 and 2007, respectively. He joined KDD Corporation (currently KDDI Corporation), Japan in 1999, and has been working at KDDI Research since 2001. He is currently a senior manager of the photonic transport network laboratory. He has been engaged in various $R \& D$ topics related to optical communications and networking. He has been working on national and international projects focusing on optical networking and communication technologies. He has authored and co-authored more than 200 papers and standardization contributions. He has also served as TPC chairs/members for international conferences (OFC, ONDM, OECC, etc.) and guest editors (IEICE, OSA, etc.).

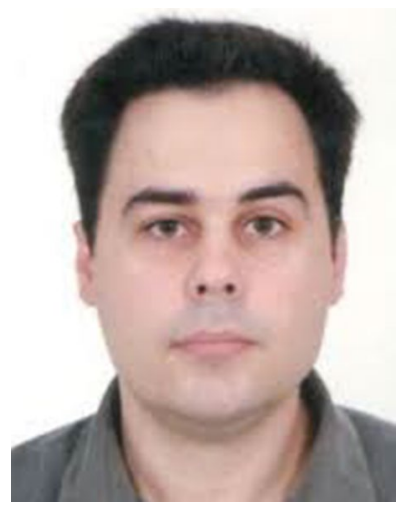

Markos Anastasopoulos is a senior researcher at the High Performance Networks Group of the University of Bristol, UK. His main research interests lie in the areas of optical and wireless communications networks, mobile and distributed computing, network design and management. He is an author/co-author of about 100 papers in peerreviewed international journal and conference proceedings. He has awarded scholarships by the Kyprianides, Eugenides, Thomaides and Propondis foundations. He is also the recipient of the best doctoral dissertation award across all engineering schools of NTUA (2009) and the best paper award at the 17th IEEE/IFIP Optical Network Design and Modeling (ONDM) conference (2013). 